\title{
Circ-ITCH sponges miR-214 to promote the osteogenic differentiation in osteoporosis via upregulating YAP1
}

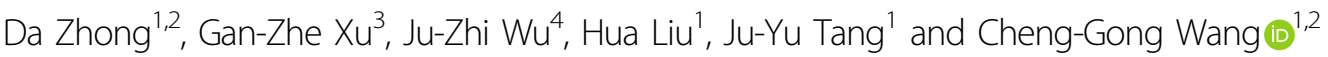

\begin{abstract}
Osteoporosis is the most prevailing primary bone disease and a growing health care burden. The aim of this study was to clarify the functional roles and mechanisms of the circ-ITCH regulating osteogenic differentiation of osteoporosis. Circ-ITCH and yes-associated protein 1 (YAP1) levels were downregulated, but the miR-214 level was upregulated in osteoporotic mice and patients. Knockdown of circ-ITCH inhibited the alkaline phosphatase (ALP) activity, mineralized nodule formation, and expression of runt-related transcription factor 2 (RUNX2), osteopontin (OPN), and osteocalcin (OCN) during osteogenic induction. Furthermore, miR-214 was a target of circ-ITCH, knockdown of miR-214 could impede the regulatory effects of sh-circ-ITCH on osteogenic differentiation. Moreover, miR-214 suppressed hBMSCS osteogenic differentiation by downregulating YAP1. Finally, in vivo experiments indicated that overexpression of circITCH could improve osteogenesis in ovariectomized mice. In conclusion, circ-ITCH upregulated YAP1 expression to promote osteogenic differentiation in osteoporosis via sponging miR-214. Circ-ITCH could act as a novel therapeutic target for osteoporosis.
\end{abstract}

\section{Introduction}

Osteoporosis is one of the most common bone diseases with bone loss and reduced bone mineral density (BMD), which increases bone fragility and occurrence of fracture ${ }^{1}$. In fact, osteoporotic fracture usually results in lasting disability and mortality in the elderly population and postmenopausal women ${ }^{2}$. In China, it is predicted that at least 90 million people suffer from osteoporosis ${ }^{3}$. Because of the high prevalence rate, there is a great demand to find effective methods of osteoporosis prevention and treatment. Studies have shown that in people with osteoporosis, bone loss is induced by the reduced bone formation and increased bone resorption ${ }^{4,5}$. The bone formation could be modulated by many transcription

\footnotetext{
Correspondence: Cheng-Gong Wang (wangchenggong@csu.edu.cn)

${ }^{1}$ Department of Orthopaedics, Xiangya Hospital of Central South University,

410008 Changsha, Hunan Province, People's Republic of China

${ }^{2}$ Hunan Key Laboratory of Aging Biology, Xiangya Hospital, Central South

University, 410008 Changsha, Hunan Province, People's Republic of China

Full list of author information is available at the end of the article

Edited by M. Agostini
}

factors and signaling pathways ${ }^{6-8}$. Therefore, researching the mechanisms that modulate bone formation will be significant for the treatment of osteoporosis.

Among the non-coding RNAs, circular RNAs (circRNAs) are highly abundant and evolutionarily conserved endogenous RNAs with a covalently closed loop ${ }^{9}$. Recent evidences have revealed that circRNAs participate in cell growth, migration, invasion, and apoptosis ${ }^{10-12}$. CircITCH is a well-known circRNA that spans several exons of itchy E3 ubiquitin-protein ligase (ITCH), it exerts inhibitory effects in many cancers through sponging certain miRNAs ${ }^{13,14}$. Recently, it was reported that during periodontal ligament stem cell (PDLSC) osteogenic differentiation, circ-ITCH was upregulated and might regulate the osteogenic differentiation through MAPK pathway $^{15}$. However, to date, the effects of circ-ITCH in osteoporosis remain largely unclear and need further investigation. Accumulating evidences suggest the critical roles of miR-214 in bone formation ${ }^{16,17}$. For example, miR-214 negatively regulated osteogenic differentiation of

\section{(c) The Author(s) 2021}

(c) (i) Open Access This article is licensed under a Creative Commons Attribution 4.0 International License, which permits use, sharing, adaptation, distribution and reproduction cc) in any medium or format, as long as you give appropriate credit to the original author(s) and the source, provide a link to the Creative Commons license, and indicate if changes were made. The images or other third party material in this article are included in the article's Creative Commons license, unless indicated otherwise in a credit line to the material. If material is not included in the article's Creative Commons license and your intended use is not permitted by statutory regulation or exceeds the permitted use, you will need to obtain permission directly from the copyright holder. To view a copy of this license, visit http://creativecommons.org/licenses/by/4.0/. 
hPDLSCs through inactivating transcription factor $4^{17}$. MiR-214 suppressed the osteogenic differentiation of bone marrow-derived mesenchymal stem cells (BMSCs) through inhibiting JNK and p38 pathways ${ }^{18}$. Furthermore, it was reported that miR-214 contained complementary sequences to circ-ITCH, and circ-ITCH served tumorsuppressive roles in gliomas by targeting miR-214 ${ }^{19}$. In addition, our bioinformatic analysis (StarBase; http:// starbase.sysu.edu.cn/index.php) showed there were binding sites between circ-ITCH and miR-214. However, it is still unknown whether miR-214 could be regulated by circ-ITCH to mediate osteoporosis progression. Thus, in this study, we hypothesize that circ-ITCH may be involved in osteoporosis and may regulate the hBMSCs osteogenic differentiation by interacting with miR-214.

Recent studies suggest that yes-associated protein 1 (YAP1), an important downstream effector of the Hippo signaling pathway, has crucial roles in cell growth, differentiation, and organ size control ${ }^{20,21}$. In particular, YAP1 has been implicated as a regulator of osteoblast differentiation. For example, upregulation of YAP1 could promote mesenchymal stem cells to differentiate into osteocytes $^{22}$. In addition, YAP1 promoted osteogenesis by interacting with $\beta$-catenin in osteoblast-lineage cells ${ }^{23}$. Moreover, knocking down YAP1 in mice using Dmp1-Cre decreased osteoblast number and bone mass ${ }^{24}$. However, the function of YAP1 on the osteogenic differentiation of hBMSCs in osteoporosis remains further investigated. Interestingly, bioinformatics analysis (StarBase) found putative YAP1 response elements on miR-214. But whether miR-214 could associate with YAP1 has never been reported. Therefore, we focus on the interaction between miR-214 and YAP1 in hBMSCs osteogenic differentiation in osteoporosis.

In this study, we firstly found that circ-ITCH was downregulated in osteoporosis but up-regulated in osteogenic differentiation of hBMSCs. We demonstrated that circITCH sponged miR-214 to indirectly up-regulate YAP1, thus promoting osteogenic differentiation in vitro and in vivo. This research may provide a new target for regulating the osteogenic ability of hBMSCs and improve the therapeutic effect for osteoporosis.

\section{Materials and methods}

\section{Clinical samples and isolation of hBMSCs}

All procedures were approved by the Medical Ethics Committee of the Xiangya Hospital, Central South University (Changsha, Hunan, China). Bone marrow samples were obtained with informed consent from 15 female postmenopausal osteoporosis patients and 15 postmenopausal women without osteoporosis recruited from Xiangya Hospital, Central South University. Osteoporosis was diagnosed using the World Health Organization parameters. Primary hBMSCs were separated from bone marrow samples as previously described ${ }^{25}$.

\section{Cell culture}

The cell line of human BMSCs and 293T cells were obtained from the American Type Culture Collection (ATCC, Manassas, VA, USA). All the cell lines included in this study have been authenticated by STR profiling and tested for mycoplasma contamination. Cells were cultured in Dulbecco's modified Eagles medium (DMEM, Gibco, Grand Island, NY, USA) supplemented with $10 \%$ fetal bovine serum (FBS, Invitrogen, Carlsbad, CA, USA), and $1 \%$ penicillin-streptomycin (Invitrogen) at $37^{\circ} \mathrm{C}$ in a humidified condition of $5 \% \mathrm{CO}_{2}$.

\section{Osteogenic differentiation}

Osteogenic differentiation assay of cultured hBMSCs was performed as described ${ }^{26}$. Briefly, hBMSCs were seeded in 24-well plates $\left(5 \times 10^{4}\right.$ cells/well $)$ and grown to $80 \%$ confluence. Then hBMSCs were treated with a medium containing $200 \mu \mathrm{M}$ ascorbic acid, $10 \mathrm{mM}$ $\beta$-glycerophosphate, and $100 \mathrm{nM}$ dexamethasone (all from Sigma, St Louis, MO, USA) for 14 days to induce osteogenic differentiation, and induction medium was replaced every 3 days.

\section{Lentiviruses infection and cell transfection}

sh-circ-ITCH, circ-ITCH overexpression (OE-circITCH) and YAP1 overexpression (OE-YAP1) lentiviruses, miR-214 mimics, miR-214 inhibitor, and their negative controls (NC) were all obtained from GenePharma (Shanghai, China). To generate lentiviruses, 293T cells were co-transferred with pGLVH1/GFP/Puro (GenePharma) and the packaging plasmids Helper 1.0 (GeneChem, Shanghai, China). Then lentiviruses were harvested and purified $72 \mathrm{~h}$ after transfection. The hBMSCs were seeded in 24-well plates to reach about $40 \%$ confluence and then infected with lentiviruses (multiplicity of infection of 50) in the presence of polybrene $(5 \mu \mathrm{g} / \mathrm{mL}$, GeneChem). Stably transfected hBMSCs were selected by $1 \mu \mathrm{g} / \mathrm{mL}$ puromycin (Invitrogen). The sequences of miR-214 mimics, miR-214 inhibitor, and their NC were as follows:

MiR-214 mimics: sense, 5'-ACAGCAGGCACAGACA GGCAG-3';

antisense, $5^{\prime}$-GCCUGUCUGUGCCUGCUGUUU- ${ }^{\prime}$. Mimics NC: sense, 5'-UUCUCCGAACGUGUCACG UTT-3';

antisense, 5'-ACGUGACACGUUCGGAGAATT- ${ }^{\prime}$. MiR-214 inhibitor: 5'-ACUGCCUGUCUGUGCCUGC UGU-3'.

Inhibitor NC: 5'-CAGUACUUUUGUGUAGUACA A-3'. 


\section{Luciferase reporter assay}

The potential binding sites between circ-ITCH and miR-214, miR-214, and YAP1 were predicted through StarBase online website (http://starbase.sysu.edu.cn/ index.php). The 3'-UTR fragment of circ-ITCH and YAP1 containing the wild-type or mutant miR-214 binding sites were synthesized and inserted into PsiCHECK-2 vectors (Promega, Madison, WI, US), named as circ-ITCH-WT, circ-ITCH-MUT, YAP1-WT, or YAP1-MUT. hBMSCs and 293T cells were cultured in 6-well plates to reach about 70\% confluence. Then cells were co-transfected with circ-ITCH-WT, circ-ITCHMUT, YAP1-WT, or YAP1-MUT vector and either miR214 mimics or miR-214 inhibitor using Lipofectamine ${ }^{\circledR}$ 2000 (Invitrogen). The luciferase activity in each group was determined on a Dual-Luciferase Assay System (Promega) after transfection $48 \mathrm{~h}$.

\section{RNA immunoprecipitation (RIP) assay}

hBMSCs and 293T cells were transfected with miR-214 inhibitor or inhibitor NC for $24 \mathrm{~h}$. RIP assay was conducted with an EZ-Magna RIPTM RNA-binding Protein Immunoprecipitation Kit (Millipore Corporation, USA). Antibodies against Ago2 (\#ab186733, 1:50, Abcam, Cambridge, UK) and IgG (\#ab205718, 1:100, Abcam) were used for RIP. Finally, the immunoprecipitated RNAs were extracted and quantified by RT-qPCR.

\section{Alizarin red $\mathrm{S}$ staining and quantification}

After osteogenic differentiation, hBMSCs were fixed using 70\% ethanol, incubated with $2 \%$ Alizarin red staining reagent (Sigma) at room temperature (RT) for 20 min. Subsequently, hBMSCs were washed using phosphate-buffered saline (PBS, Invitrogen), and visualized using a light microscope (Zeiss, Germany). The accessing process was conducted by an assessor blind to treatment allocation. For Alizarin Red S quantification, the stains were dissolved in $1 \mathrm{~mL}$ cetylpyridinium chloride buffer (Sigma) for $1 \mathrm{~h}$ and the absorbance was measured at $562 \mathrm{~nm}$. The level of mineralization was calculated based on the Alizarin red S standard curve and represented as $\mu \mathrm{mol} / \mu \mathrm{g}$ protein.

\section{Alkaline phosphatase (ALP) staining and quantification}

After osteogenic differentiation, hBMSCs were fixed using 4\% PFA and stained using an ALP staining Kit (\#RE051, GeFan Biotechnology Technology, shanghai, China). After washing with PBS, stains were visualized with a microscope (Zeiss, Germany). The accessing process was conducted by an assessor blind to treatment allocation. For ALP activity assay, hBMSCs were lysed by 1\% Triton X-100 (Sigma) for 15 min, the supernatant was collected through centrifugation $(14,000 \times g, 5 \mathrm{~min})$, ALP activity was measured by an ALP Colorimetric Assay Kit
(BioVision, Milpitas, CA, USA) on a microplate reader at $405 \mathrm{~nm}$.

\section{Ovariectomy (OVX) animal model}

Sixty healthy 8-week-old C57BL/J6 female mice were obtained from SLAC Laboratory Animal Company, Ltd. (Shanghai, China) and housed in standard pathogen-free conditions. Mice were randomly divided into the following groups: Sham, OVX, OVX + OE-NC, and OVX + OE-circ-ITCH groups. After 1 week of acclimatization, mice were anesthetized with $5 \%$ ketamine and underwent bilateral OVX or sham operation in a bioclean environment as previously reported ${ }^{27}$. One week after OVX surgery, circ-ITCH overexpression lentiviral vector $(\mathrm{OE}$-circ-ITCH) or negative control vector (OE$\mathrm{NC}$ ) was injected into OVX mice through tail intravenous injection once per week for 12 weeks. After 13 weeks of OVX surgery, the femurs of mice were harvested to measure the degree level of osteoporosis. BMD was measured using Dual-energy X-ray absorptiometry (Hologic, Bedford, MA, USA). The accessing process was conducted by an assessor blind to treatment allocation. All procedures were approved by the Institutional Animal Care and Use Committee of the Xiangya Hospital, Central South University (Changsha, Hunan, China).

\section{Hematoxylin and eosin (H\&E) staining and Alcian blue staining}

Femur samples from mice were fixed in 4\% PFA overnight and decalcified in 10\% ethylenediamine tetra-acetic acid for 30 days. Then paraffin-embedded samples were sectioned into 5 - $\mu \mathrm{m}$-thick slices. Then slices were treated with gradient ethanol and stained by H\&E (Nanjing Jiancheng Bioengineering Institute, Nanjing, Jiangsu, China) or $1 \%$ Alcian Blue (Abcam) at RT. Images from each group were photographed with a microscope (Zeiss, Germany). The accessing process was conducted by an assessor blind to treatment allocation.

\section{Reverse transcription-quantitative polymerase chain reaction (RT-qPCR)}

Total RNA was isolated from bone marrow samples and hBMSCs with TRIzol Reagent (Invitrogen). Then firststrand cDNA synthesis was produced using the PrimeScript RT reagent Kit (for mRNAs, Takara, Dalian, China) or TaqMan MicroRNA Reverse Transcription Kit (for miR-214, ThermoFisher Scientific). The qPCR was conducted with the SYBR Premix EX Taq Kit (Takara) on an ABI 7500HT real-time PCR system. Glyceraldehyde-3phosphate dehydrogenase (GAPDH) or U6 small nuclear RNA (U6 snRNA) was used as a control for mRNA or miRNA, respectively. The expression levels were measured through the $2^{-\Delta \Delta C t}$ method. Primer sequences were 
synthesized by Sangon Biotech (Shanghai, China) as follows:

Circ-ITCH F: 5'-GCAGAGGCCAACACTGGAA-3'; Circ-ITCH R: $5^{\prime}$-TCCTTGAAGCTGACTACGCTG AG-3';

MiR-214 F: 5'-TGCCTGTCTACACTTGCTGTGC-3', MiR-214 R: 5'-GCGAGCACAGAATTAATACGAC-3'; YAP1 F: 5'-TTCGGCAGGCAATACGGAAT-3', YAP1 R: 5'-GTTGAGGAAGTCGTCTGGGG-3'; Osteocalcin (OCN) F: 5'-GGCAGCGAGGTAGTGAAGAG-3',

OCN R: 5'-CTAGACCGGGCCGTAGAAG-3'; Osteopontin (OPN) F: 5'-GATGGCCGAGGTGATA GTGT-3',

OPN R: 5'-GTGGGTTTCAGCACTCTGGT-3'; RUNX2 F: 5'-CGGAATGCCTCTGCTGTTAT-3', RUNX2 R: 5'-TTCCCGAGGTCCATCTACTG-3'; GAPDH R: $5^{\prime}$-CCAGGTGGTCTCCTCTGA-3', GADPH F: 5'-GCTGTAGCCAAATCGTTGT-3'; U6 R: 5'-CTCGCTTCGGCAGCACA-3'; U6 F: 5'-AACGCTTCACGAATTTGCGT-3'.

\section{Western blot assay}

Total protein was isolated from hBMSCs with RIPA lysis buffer (Beyotime, Shanghai, China) containing proteinase inhibitors (Sigma). Protein concentration was detected with an Enhanced BCA Protein Assay Kit (Beyotime). $20 \mu \mathrm{g}$ total proteins were electrophoresed with 10\% SDS-PAGE and then transferred onto PVDF membranes (Millipore, Burlington, MA, USA). After blocking by $5 \%$ non-fat milk for $2 \mathrm{~h}$ at RT, membranes were incubated with respective primary antibodies against YAP1 (13584-1-AP, 1:2000, Proteintech), RUNX2 (ab76956, 1:1000, Abcam), OPN (ab8448, 1:1500, Abcam), OCN (ab93876, 1:1000, Abcam) and GAPDH (ab181602, 1:5000, Abcam) at $4{ }^{\circ} \mathrm{C}$ overnight. Finally, membranes were incubated with the appropriate secondary HRP antibodies (ab7090 or ab190475, 1:5000, Abcam) for $2 \mathrm{~h}$ at RT. The membranes were exposed with chemiluminescence substance (ThermoFisher Scientific) and band intensity was measured by ImageJ software.

\section{Statistical analysis}

All experiments have been conducted independently at least three times. All the data meet the assumption of normal distribution. Data were analyzed with Prism 6.0 (GraphPad Software, USA) and expressed as mean \pm standard deviation (SD). Statistical analysis between two groups was performed using the Student's $t$-test and multiple comparisons were carried out using one-way analysis of variance (ANOVA) followed by Tukey's post hoc test. $P<0.05$ was considered statistically significant.

\section{Results}

Circ-ITCH and YAP1 expressions were upregulated and miR-214 expression was downregulated during osteogenic differentiation

After induction for 0,7 , and 14 days, the osteogenic capabilities of hBMSCs were evaluated by ALP staining and ALP activity assay for osteoblast differentiation, Alizarin red $\mathrm{S}$ staining, and quantification for calcium mineralization. We observed increased ALP staining (Fig. 1A, scale bar: $100 \mu \mathrm{m}$ ) and elevated ALP activity (Fig. 1B), as well as significantly higher levels of mineralized nodule formation (Fig. 1C, D, scale bar: $200 \mu \mathrm{m}$ ) at day 7 and 14, suggesting successful induction of osteogenic differentiation. Furthermore, we measured the expression of RUNX2, OPN, and OCN in hBMSCs. The mRNA (Fig. $1 \mathrm{E}$ ) and protein (Fig. 1F) levels of these osteogenic markers were increased consistently throughout the differentiation process. Moreover, circ-ITCH expression level and YAP1 mRNA and protein levels were gradually increased, but the miR-214 expression was gradually reduced in a time-dependent manner during the osteogenic differentiation (Fig. 1G, H). Additionally, we measured the levels of circ-ITCH, miR-214, and YAP1 in control samples and osteoporotic samples. Circ-ITCH and YAP1 expressions were downregulated whereas miR214 expression was higher in osteoporotic samples (Fig. 1I). These data hinted that circ-ITCH, miR-214, and YAP1 may be involved in osteogenic differentiation of osteoporosis.

\section{Circ-ITCH promoted the osteogenic differentiation of hBMSCs in vitro}

To examine the roles of circ-ITCH in osteogenic differentiation, lentiviruses infection in hBMSCs was performed to overexpress or knockdown its expression, respectively. The transfection efficiency was determined through RT-qPCR, circ-ITCH expression was markedly increased with transfection of OE-circ-ITCH and decreased with transfection of sh-circ-ITCH compared to their negative controls (Fig. 2A). The ALP activity (Fig. 2B) and ALP staining (Fig. 2D, scale bar: $100 \mu \mathrm{m})$, and mineralized nodule formation (Fig. 2C, E, scale bar: $200 \mu \mathrm{m}$ ) were significantly elevated by overexpression of circ-ITCH, whereas suppressed by knockdown of circ-ITCH after osteogenic differentiation for 14 days. Besides, the mRNA (Fig. 2F) and protein (Fig. 2G) levels of osteogenic markers RUNX2, OPN, and OCN were all induced by upregulating circ-ITCH, whereas were decreased by knocking down circ-ITCH. These results confirmed that circITCH promoted the osteogenic differentiation of hBMSCs in vitro. 


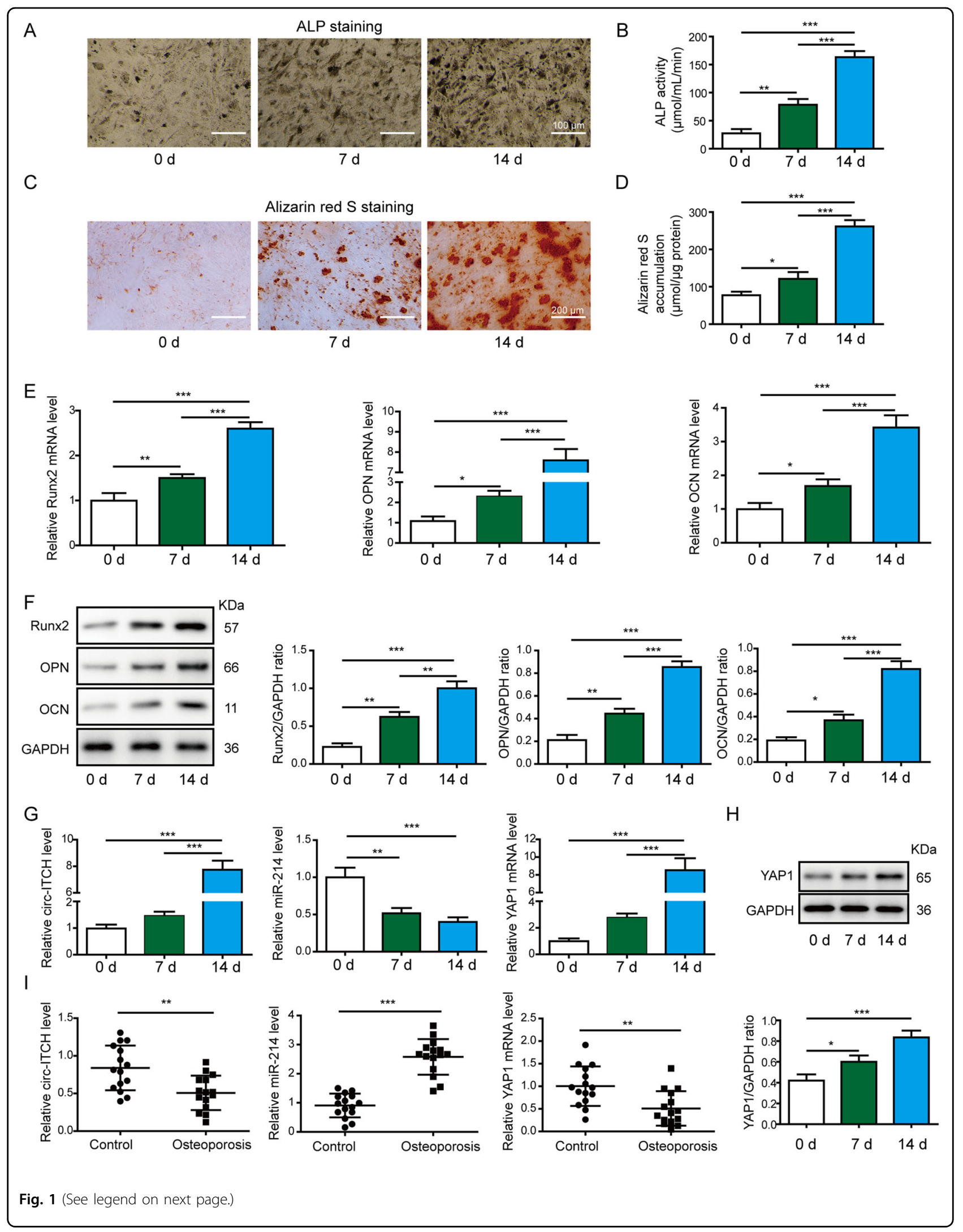


(see figure on previous page)

Fig. 1 Circ-ITCH and YAP1 expressions were upregulated and miR-214 expression was downregulated during hBMSCs osteogenic differentiation. Osteogenic differentiation of hBMSCs was evaluated by ALP staining (A Scale bar: $100 \mu \mathrm{m}$ ) and ALP activity assay (B) at 0, 7, and 14 days after osteogenic induction. C, D Calcium deposition determined by Alizarin red S staining (Scale bar: $200 \mu \mathrm{m}$ ) and accumulation of mineralization on days 0,7 , and 14 after osteogenic induction. The mRNA and protein levels of osteogenic genes RUNX2, OPN, and OCN were determined by RT-qPCR (E) and western blot assay $(\mathbf{F})$ at 0, 7, and 14 days. G The expression levels of circ-ITCH, miR-214, and YAP1 were determined by RT-qPCR after 0,7 , and 14 days of osteogenic culture. $\mathbf{H}$ The protein levels of YAP1 were determined by western blot assay after 0,7 , and 14 days of osteogenic culture. I Relative Circ-ITCH, miR-214, and YAP1 levels in 15 control (non-osteoporotic) samples and 15 osteoporotic samples. ${ }^{*} P<0.05$, ${ }^{*} P<0.01$, and ${ }^{* *} P<0.001$.

\section{Circ-ITCH directly targeted and suppressed miR-214 expression}

The underlying mechanisms of circ-ITCH-mediated osteogenic differentiation were then explored. Overexpressing circ-ITCH decreased miR-214 while knocking down it increased miR-214 expression in hBMSCs (Fig. 3A). In addition, bioinformatics analysis (StarBase; http:// starbase.sysu.edu.cn/index.php) by StarBase online website showed that the complementary sites between circITCH and miR-214 (Fig. 3B). Then the targeted relationship between circ-ITCH and miR-214 was validated through a dual-luciferase reporter and RIP assays. The luciferase activity of circ-ITCH-WT was significantly lower when transfected with miR-214 mimics, but higher when transfected with miR-214 inhibitor (Fig. 3C). But, no significant changes were found in circ-ITCH-MUT groups (Fig. 3C). Also, circ-ITCH was specifically enriched in the Ago2 immunoprecipitates compared with control IgG (Fig. 3D). Moreover, miR-214 knockdown decreased the enrichment of circ-ITCH to Ago2 (Fig. 3D). These data revealed that circ-ITCH directly targeted and suppressed miR-214 expression.

\section{Circ-ITCH induced the osteogenic differentiation through directly targeting miR-214}

We further explored whether circ-ITCH regulated the osteogenic differentiation via targeting miR-214. The hBMSCs were transfected with sh-circ-ITCH vector alone or co-transfected with miR-214 inhibitor, then circ-ITCH and miR-214 expressions were measured. As shown in Fig. 4A, B, sh-circ-ITCH transfection downregulated circ-ITCH expression and upregulated miR-214 expression, whereas co-silencing miR-214 downregulated miR-214 expression and had no effect on circ-ITCH expression. Functional studies showed that knockdown of miR-214 in hBMSCs could reverse the effects of sh-circ-ITCH on ALP staining (Fig. 4C, scale bar: $100 \mu \mathrm{m}$ ), ALP activity (Fig. 4E), and mineralized nodule formation (Fig. 4D, F, scale bar: $200 \mu \mathrm{m}$ ). Furthermore, the mRNA (Fig. 4G) and protein (Fig. 4H) levels of osteogenic markers RUNX2, OPN, and OCN were also reinforced by miR-214 inhibitor co-transfection compared to sh-circ-ITCH transfection alone. Taken together, circ-ITCH positively regulated the osteogenic differentiation of hBMSCs via directly targeting miR-214.

\section{MiR-214 directly and negatively regulated YAP1 expression}

Previous results indicated that YAP1 was an important modulator of osteogenic differentiation ${ }^{28}$. Next, we investigated whether YAP1 could be regulated by miR214. After transfection with miR-214 mimics or inhibitors, the expression levels of miR-214 and YAP1 in hBMSCs were further detected. It was shown that the level of miR214 was higher in miR-214 mimics group and lower in the miR-214 inhibitor group (Fig. 5A). The mRNA and protein levels of YAP1 were dramatically attenuated in the miR214 mimics group, whereas their expression levels were obviously increased in the miR-214 inhibitor group (Fig. $5 \mathrm{~B}, \mathrm{C})$. Then, the putative binding sites between miR-214 and YAP1 were predicted using StarBase (Fig. 5D). To further corroborate the specific interaction between miR214 and YAP1, a dual-luciferase reporter assay was conducted. The luciferase activity in the YAP1-WT group was reduced by miR-214 mimics and increased by the miR-214 inhibitor, but no differences were revealed in the YAP1MUT groups (Fig. 5E). Taken together, these data suggested that miR-214 was a negative regulator of YAP1.

\section{MiR-214 inhibited osteogenic differentiation through downregulating YAP1}

To verify the roles of miR-214 on osteogenic differentiation and involvement of YAP1, hBMSCs were cotransfected with miR-214 mimics and the OE-YAP1 vector. As shown in Fig. 6A, C, miR-214 mimics downregulated the mRNA and protein levels of YAP1, while cotransfection of the OE-YAP1 vector upregulated the expression of YAP1, but had no effect on the miR-214 level. The ALP staining (Fig. 6D, scale bar: $100 \mu \mathrm{m}$ ), ALP activity (Fig. 6F), and mineralized nodule formation (Fig. $6 \mathrm{E}, \mathrm{G}$, scale bar: $200 \mu \mathrm{m}$ ) in hBMSCs were significantly decreased by overexpression of miR-214. However, YAP1 overexpression rescued the above effects induced by miR214 mimics. Moreover, miR-214 overexpression also inhibited RUNX2, OPN, and OCN mRNA (Fig. 6H) and protein (Fig. 6I) levels. Furthermore, inhibited osteogenic markers mediated by miR-214 were significantly attenuated by OE-YAP1, as indicated by the upregulation of RUNX2, OPN, and OCN expression at both mRNA and protein levels (Fig. 6H, I). These results implied that miR- 


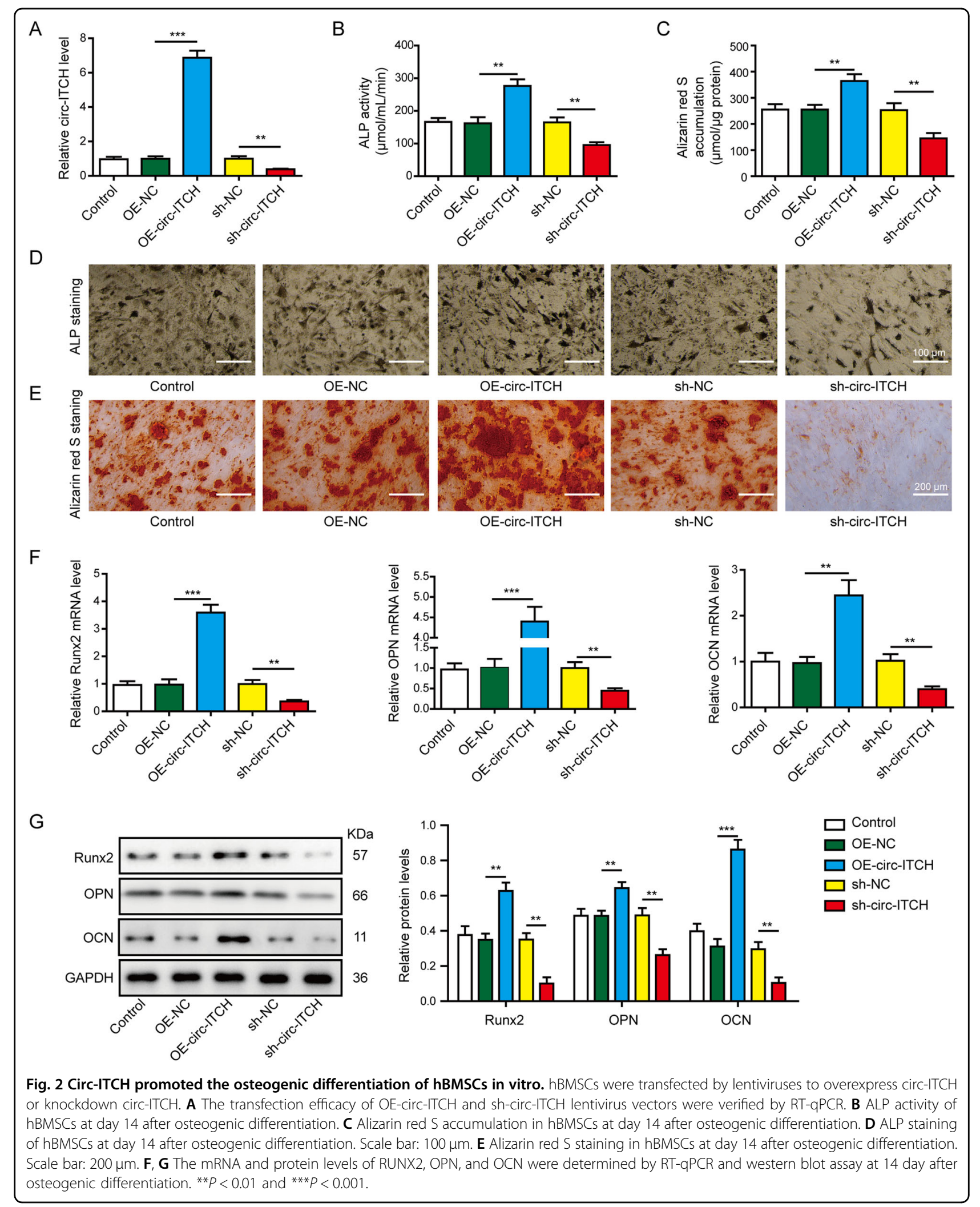




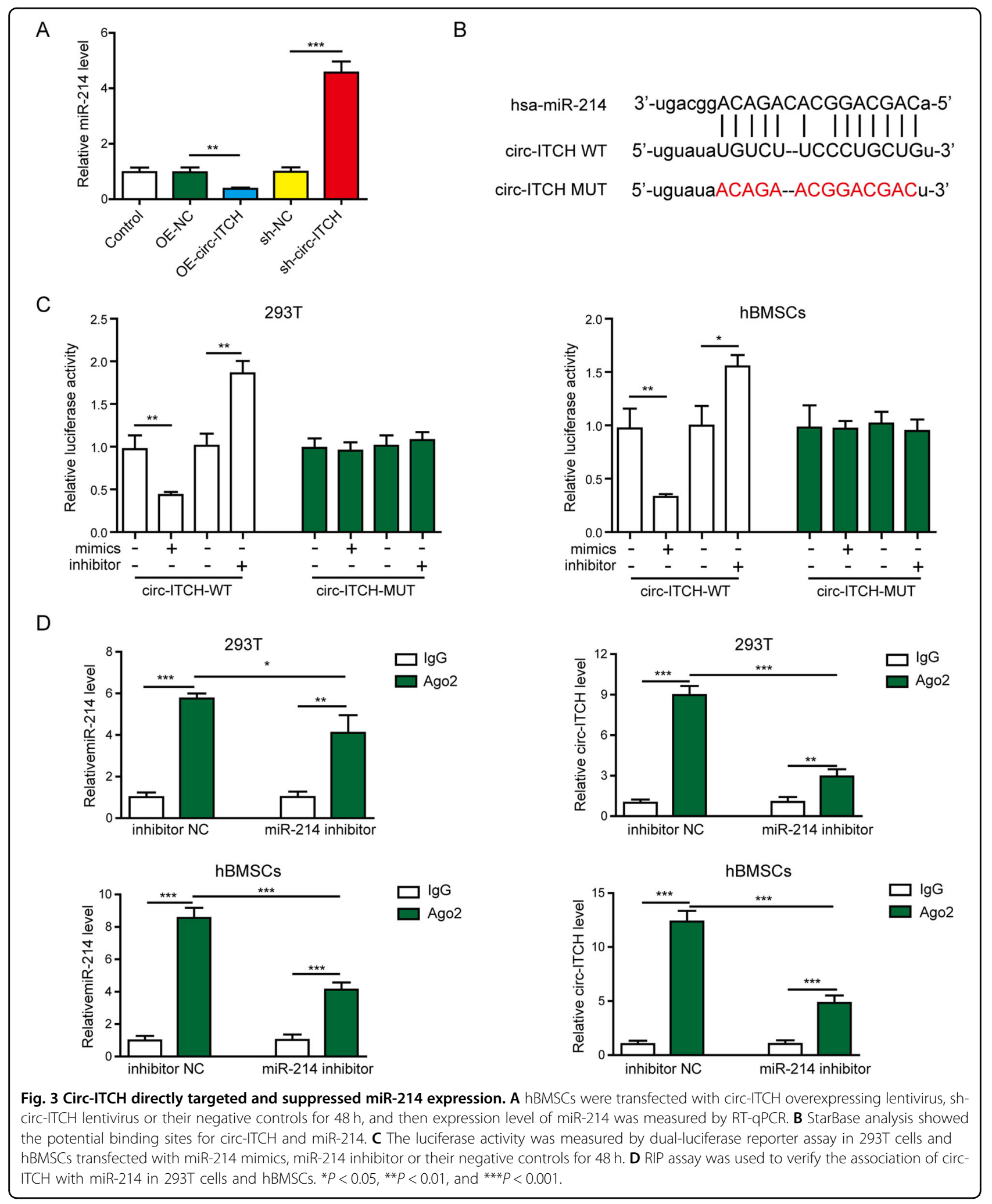




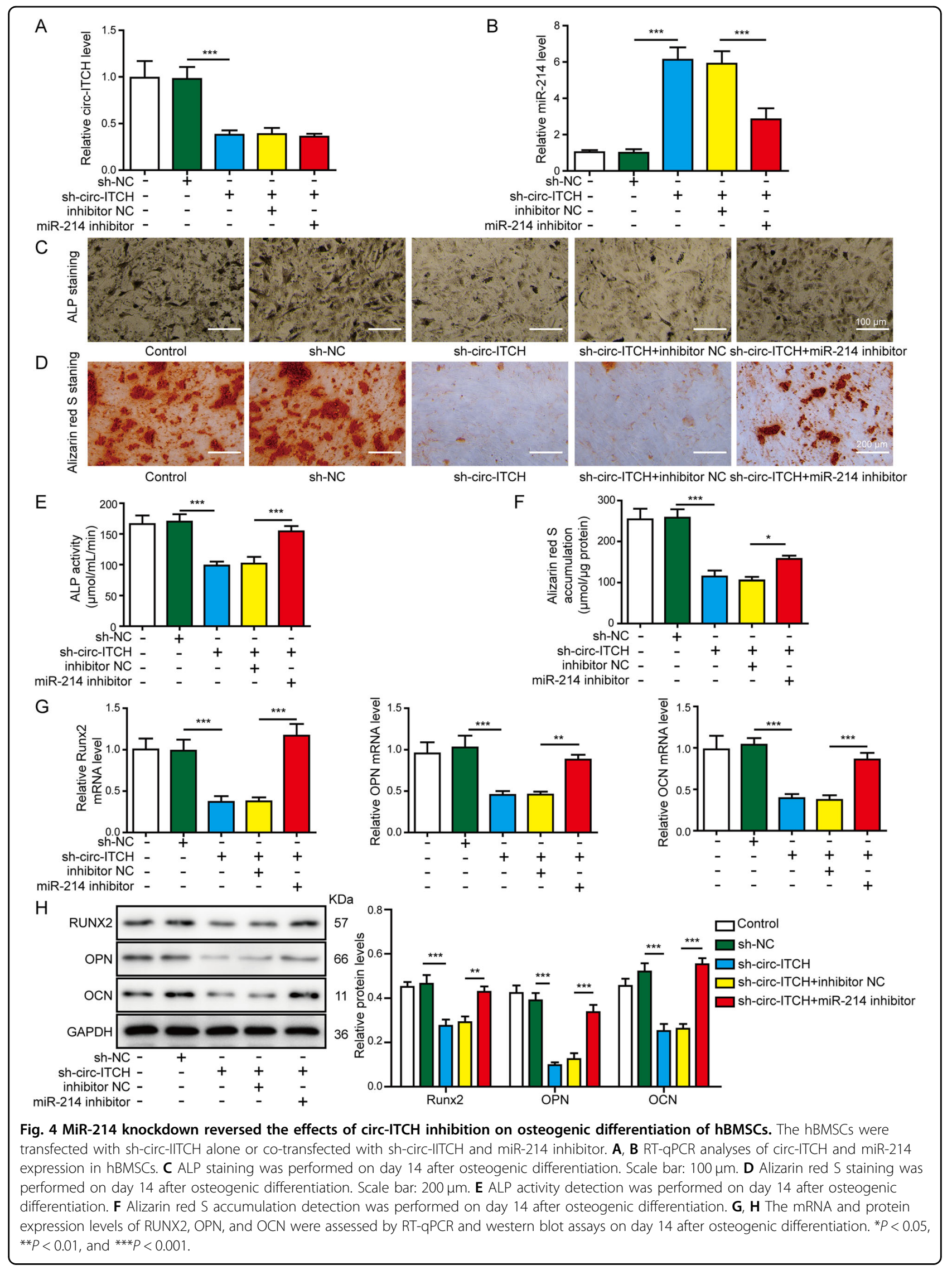




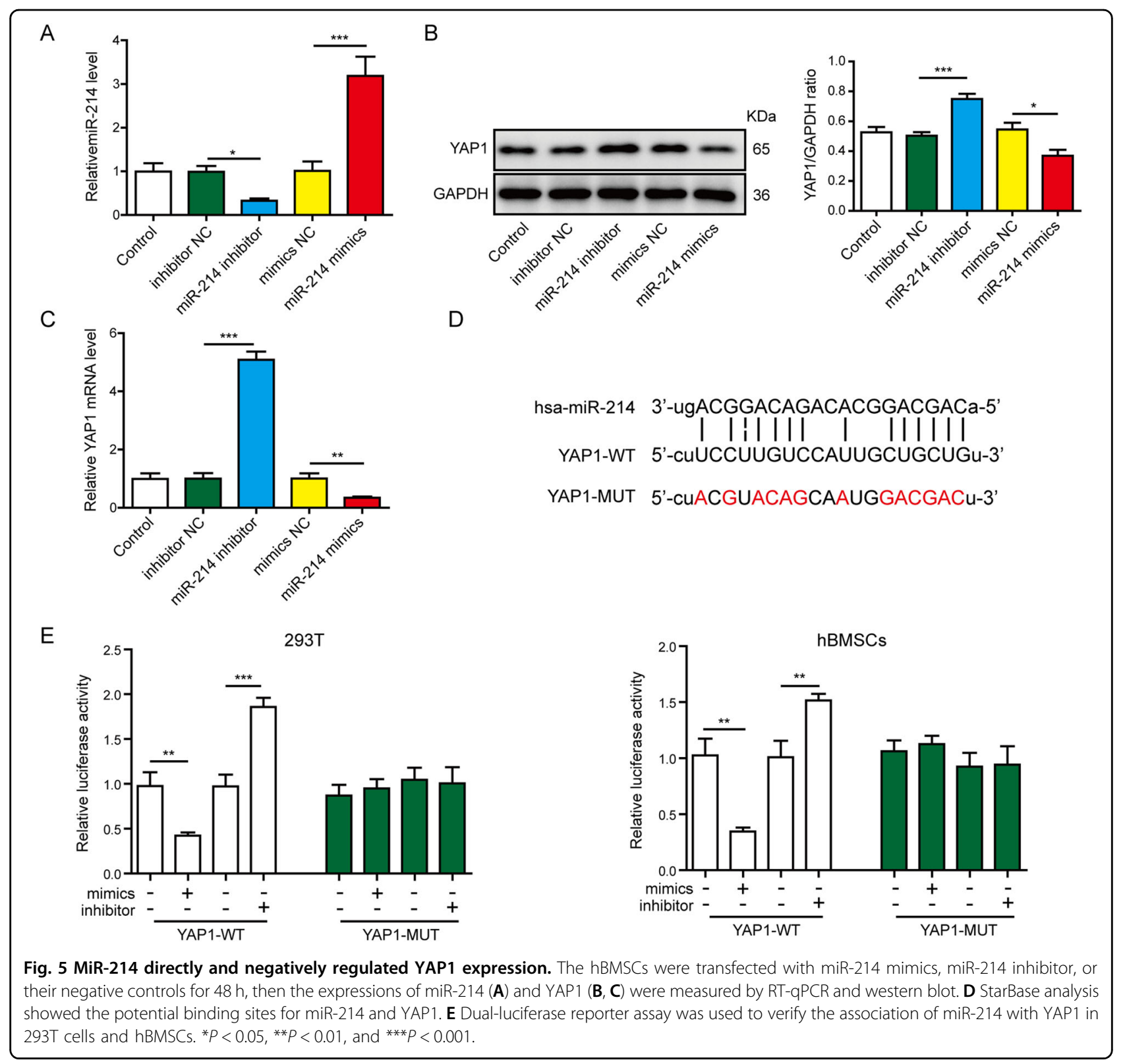

214 negatively regulated hBMSCs osteogenic differentiation by functionally targeting and inhibiting YAP1.

\section{Circ-ITCH overexpression alleviated the symptoms of osteoporosis in vivo}

To determine whether circ-ITCH has the capability to ameliorate osteoporosis in vivo, we injected OE-ITCH or OE-NC lentiviral vector into OVX mice. Compared with the sham group, the OVX group showed the damaged bone tissue structures (Fig. 7A, scale bar: $200 \mu \mathrm{m}$ ) and apparent bone loss with decreased BMD (Fig. 7B), which indicated the model of the osteoporotic mice was successfully established. OVX-associated changes in the histological morphology were restored by circ-ITCH overexpression, showing the improved bone tissue structures (Fig. 7A, scale bar: $200 \mu \mathrm{m}$ ) and the increased BMD (Fig. 7B). The mRNA levels of RUNX2, OPN, and OCN were lower in the OVX group, while were upregulated by circ-ITCH overexpression compared with OVX and OVX + OE-NC groups (Fig. 7C). Moreover, OVX mice showed the decreased circ-ITCH and YAP1 expression levels and increased miR-214 expression (Fig. 7D). However, OVX mice injected with OE-circ-ITCH lentiviral vector indicated the increased expression of circ-ITCH and YAP1 while decreased miR-214 expression compared with OVX and OVX + OE-NC mice (Fig. 7D). Taken together, these results demonstrated circ-ITCH overexpression could prevent osteoporosis progression in vivo. 


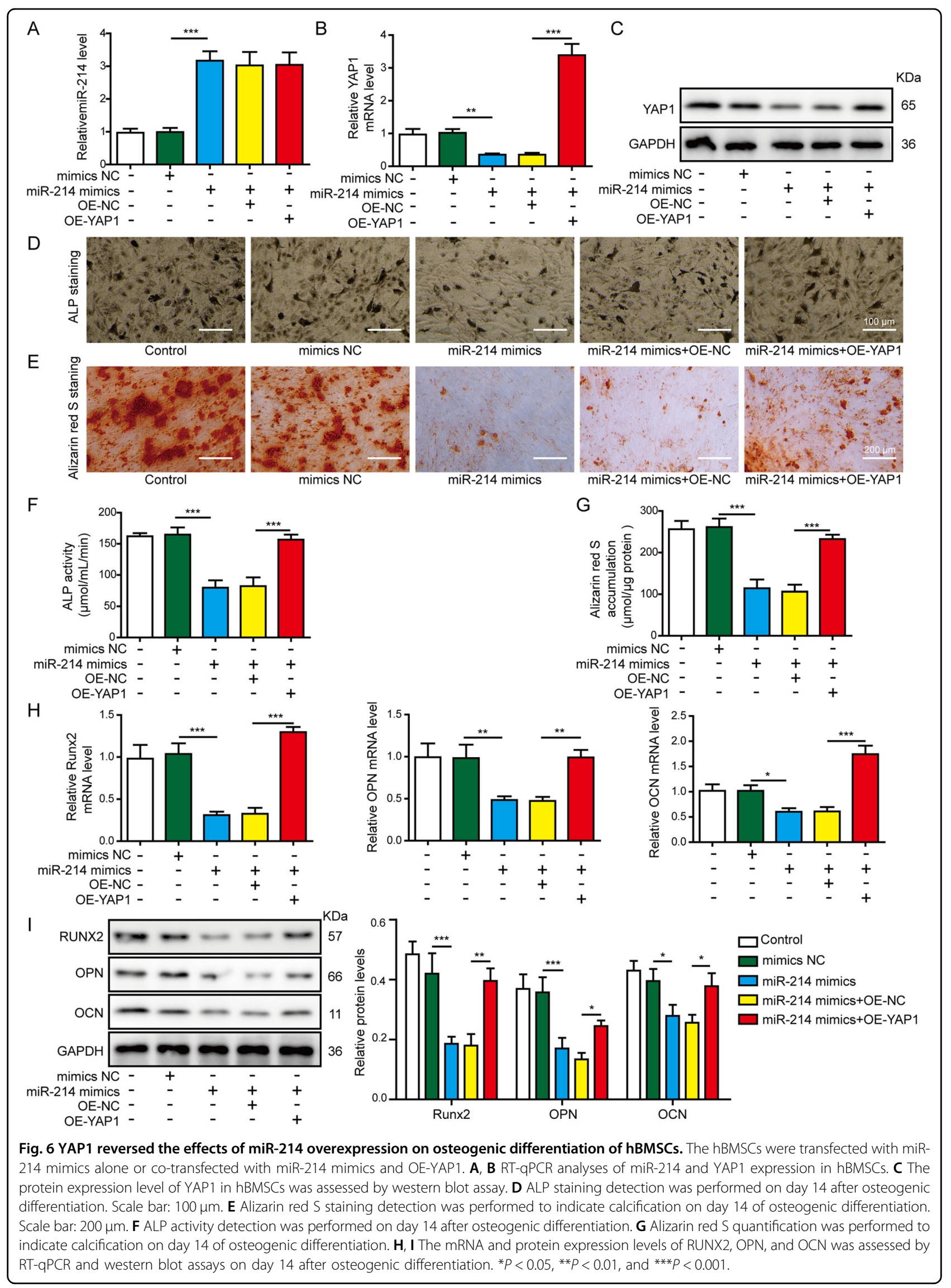




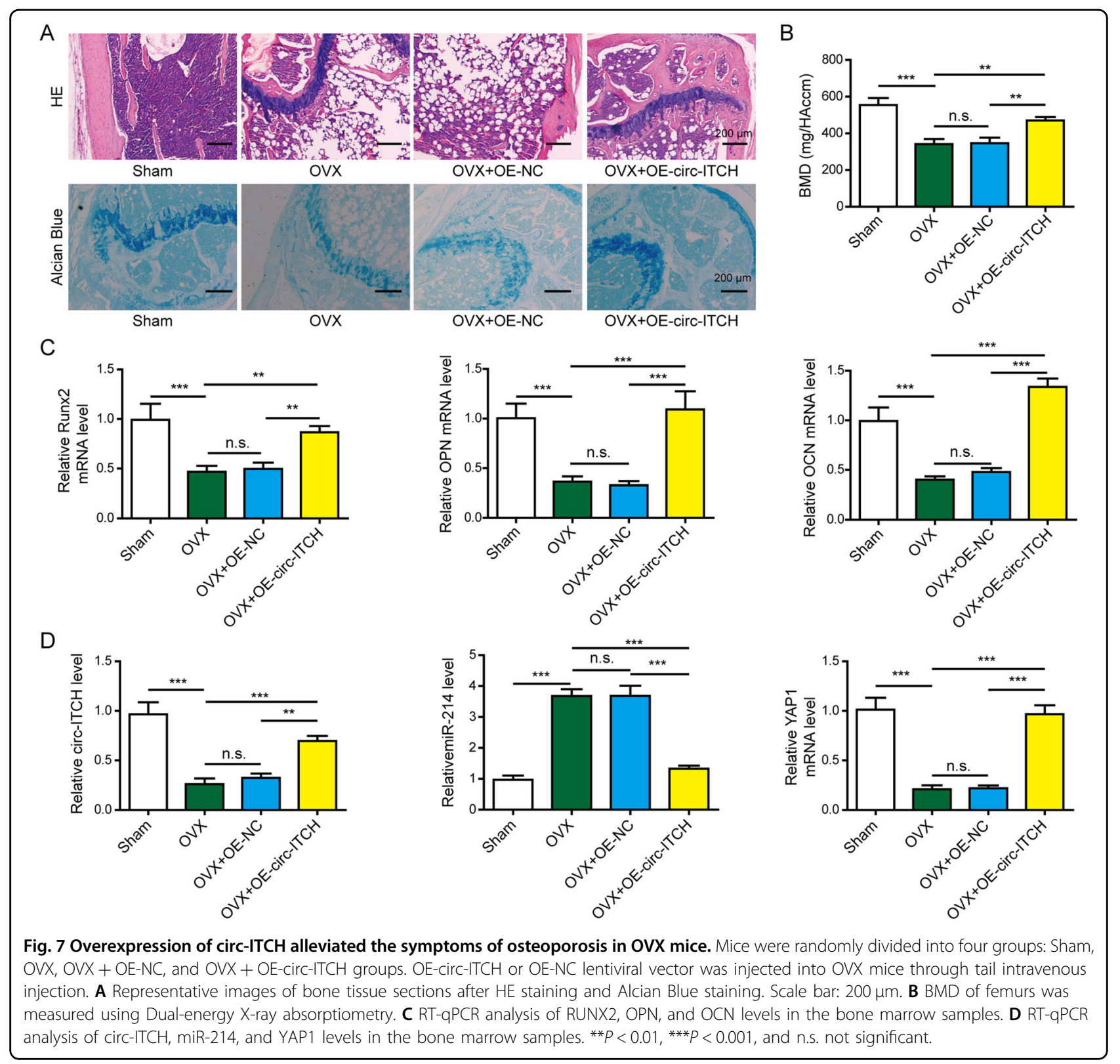

\section{Discussion}

Osteoporosis is still a major medical challenge worldwide. In recent years, tissue regeneration through the differentiation of MSCs has been a prospective therapeutic approach to treat osteoporosis ${ }^{29}$. hBMSCs are typical adult MSCs with self-renew capabilities and can differentiate into osteoblasts, which may be a potential cell source for bone tissue engineering ${ }^{30}$. The osteogenic differentiation of hBMSCs is vital for the application for bone regeneration $^{31}$. Therefore, it is important to study the molecular mechanisms that control hBMSCs osteogenic differentiation. We demonstrated that overexpression of circ-ITCH effectively promoted hBMSCs osteogenic differentiation and prevented osteoporosis in the OVX mice model by targeting the miR-214/YAP1 axis. Our results provided new insight into the expression, function, and mechanism of circ-ITCH in osteogenic differentiation of osteoporosis.

Non-coding RNAs are important post-transcriptional regulators associated with the regulation of bone formation $^{32,33}$. Previous studies have identified many circRNAs as biomarkers for osteoporosis, such as circ-RUNX2, circ19142, and circ-5846 $6^{34-36}$. For example, circRNA-28313 promoted OVX-induced bone resorption in mice by modulating miR-195a/CSF1 axis ${ }^{33}$. However, circRNA0074834 could promote BMSCs osteogenic differentiation through sponging miR-942-5 $\mathrm{p}^{34}$. Recently, many studies have verified that circ-ITCH has essential roles in the tumorigenesis and progression of human cancers $^{37}$. For 
example, circ-ITCH reduced SP-1 expression via the PTEN/PI3K/AKT pathway, which suppressed proliferation, migration, and invasion of osteosarcoma cells ${ }^{38}$. Moreover, circ-ITCH might interact with miR-34a and miR-146a to regulate PDLSC osteogenic differentiation via the MAPK pathway ${ }^{15}$. These studies suggested that circ-ITCH might have clinical significance in bone regeneration and bone-related diseases. However, their regulatory functions and mechanisms need to be further investigated. In this study, we aimed at investigating the expression and function of circ-ITCH in osteoporosis. We observed that circ-ITCH was downregulated in bone marrow samples isolated from osteoporosis patients and OVX mice. Furthermore, circ-ITCH overexpression stimulated hBMSCs osteogenic differentiation in vitro and partially attenuated the bone loss of femurs and increased $B M D$ values of OVX mice, suggesting that upregulation of circ-ITCH could prevent osteoporosis. Our results highlighted the potential of circ-ITCH in osteogenic differentiation and could potentially protect patients from osteoporosis. This is the first study reporting the expression level and role of circ-ITCH in osteoporosis.

Accumulating evidence suggested that miR-214 was implicated in osteogenic differentiation ${ }^{39,40}$. For example, miR-214 inhibited osteogenic differentiation of human PDLSCs by targeting ATF4 and suppressed BMSCs osteogenic differentiation through suppressing the JNK and $\mathrm{p} 38$ pathways ${ }^{17,18}$. Consistent with previous reports, we found that miR-214 level was higher in osteoporosis samples and overexpression of miR-214 inhibited hBMSCs osteogenic differentiation. We further researched the circ-ITCH-related molecular mechanisms during the regulation of hBMSCs osteogenic differentiation. Recently, circRNAs are recognized as miRNA sponges, reducing miRNA binding to its targets, to regulate biological processes. For example, circRNA-101368 regulated the migration of hepatocellular carcinoma via sponging miR-200a ${ }^{41}$. CircRNA-CBL.11 inhibited cell proliferation through targeting miR-6778-5p in colorectal cancer ${ }^{42}$. Moreover, circRNA-CDR1as promoted osteoblastic differentiation of periodontal ligament stem cells through the miR-7/GDF5/SMAD axis ${ }^{19}$. A recent study indicated that circ-ITCH suppressed glioma cancer through sponging miR-214 ${ }^{19}$. Consistent with previous reports, we identified that circ-ITCH showed complementary sequences to miR-214, and validated the interaction between circ-ITCH and miR-214 using luciferase reporter and RIP assays. We also demonstrated that the effects of sh-circ-ITCH on hBMSCs osteogenic differentiation were attenuated by miR-214 inhibition. This is the first research reporting circ-ITCH could sponge miR-214 to promote osteogenic differentiation in osteoporosis.

MiRNAs have been reported to regulate numerous biological processes through binding to target mRNAs and silencing the gene expression ${ }^{43}$. YAP1, as a major downstream effector of the Hippo pathway, had a vital role in the adipo-osteogenic differentiation of human $\mathrm{MSCs}^{44}$. It could also promote osteogenic differentiation of MSCs and osteoblast-lineage cells ${ }^{22,23}$. In this study, we found YAP1 expression was downregulated in osteoporosis specimens and upregulated in hBMSCs during osteogenic differentiation. Furthermore, YAP1 was a target of miR-214, and the inhibitory effects of miR-214 on hBMSCs osteogenic differentiation could be reversed by YAP1 overexpression. This is the first study to demonstrate that miR-214 suppresses the osteogenic differentiation of hBMSCs by downregulating YAP1 expression.

In conclusion, the current findings provided evidences that circ-ITCH promoted osteogenic differentiation and alleviated symptoms of osteoporosis in the OVX mice model by sponging miR-214 to upregulate YAP1 expression. Our results revealed a new mechanism of osteogenic differentiation, and further provided a novel therapeutic strategy for treating osteoporosis. However, more research is required to clarify the upstream regulatory mechanism of how circ-ITCH downregulated in osteoporosis. We will also keep working to prove whether circITCH regulates other bone degeneration diseases, such as osteoarthritis and bone senescence.

\section{Author details \\ 'Department of Orthopaedics, Xiangya Hospital of Central South University, 410008 Changsha, Hunan Province, People's Republic of China. ${ }^{2}$ Hunan Key Laboratory of Aging Biology, Xiangya Hospital, Central South University, 410008 Changsha, Hunan Province, People's Republic of China. ${ }^{3}$ Shanghai Medical College of Fudan University, 200000 Shanghai, People's Republic of China. ${ }^{4}$ Changsha Medical University, 410008 Changsha, Hunan Province, People's Republic of China}

\section{Author contributions}

C.-G.W. and D.Z. made contributions to conception and design, acquisition of data, analysis, and interpretation of data, and drafted the article. G.-Z.X., J.-Z.W., H.L., and J.-Y.T. made contributions to the acquisition of data, analysis, and interpretation of data, revised the article. All the authors approved the final version to be published.

\section{Funding}

This work was supported by the National Natural Science Foundation of China (81902308), Natural Science Foundation of Hunan Province (2020JJ5924), and National Natural Science Foundation of China (81974360).

\section{Conflict of interest}

The authors declare no competing interests.

\section{Ethics statement}

All procedures were approved by the Medical Ethics Committee of the Xiangya Hospital, Central South University (Changsha, Hunan, China). All patients were informed of the study and signed the written consent. All animal experiments and protocols have been reviewed and approved by the Institutional Animal Care and Use Committee of the Xiangya Hospital, Central South University (Changsha, Hunan, China). 


\section{Publisher's note}

Springer Nature remains neutral with regard to jurisdictional claims in published maps and institutional affiliations.

Received: 22 October 2020 Revised: 23 February 2021 Accepted: 25 February 2021

Published online: 01 April 2021

\section{References}

1. Chen, X. et al. Long non-coding RNA XIST promotes osteoporosis through inhibiting bone marrow mesenchymal stem cell differentiation. Exp. Ther. Med. 17, 803-811 (2019).

2. Sun, $X$. et al. Current progress on microRNA-based gene delivery in the treatment of osteoporosis and osteoporotic fracture. Int J. Endocrinol. 2019 6782653 (2019)

3. Jin, T. et al. Osteoporosis and renal dysfunction in a general population exposed to cadmium in China. Environ. Res. 96, 353-359 (2004).

4. Wu, Q. Y. et al. Long non-coding RNAs: a new regulatory code for osteoporosis. Front. Endocrinol. 9, 587 (2018).

5. Appelman-Dijkstra, N. M. \& Papapoulos, S. E. Modulating bone resorption and bone formation in opposite directions in the treatment of postmenopausal osteoporosis. Drugs 75, 1049-1058 (2015).

6. Zhu, Y. et al. Pharmacological activation of TAZ enhances osteogenic differentiation and bone formation of adipose-derived stem cells. Stem Cell Res. Ther. 9, 53 (2018).

7. Liang, C. et al. Inhibition of osteoblastic Smurf1 promotes bone formation in mouse models of distinctive age-related osteoporosis. Nat. Commun. 9, 3428 (2018).

8. Shen, G. et al. Foxf1 knockdown promotes BMSC osteogenesis in part by activating the Wnt/beta-catenin signalling pathway and prevents ovariectomy-induced bone loss. EBioMedicine 52, 102626 (2020).

9. Memczak, S. et al. Circular RNAs are a large class of animal RNAs with regulatory potency. Nature 495, 333-338 (2013).

10. Wang, Y., Lu, T., Wang, Q., Liu, J. \& Jiao, W. Circular RNAs: crucial regulators in the human body (review). Oncol. Rep. 40, 3119-3135 (2018).

11. Wang, Y. et al. CircRNA_014511 affects the radiosensitivity of bone marrow mesenchymal stem cells by binding to miR-29b-2-5p. Bosn. J. Basic Med. Sci. 19, 155-163 (2019).

12. Bai, N. et al. circFBLIM1 act as a ceRNA to promote hepatocellular cancer progression by sponging miR-346. J. Exp. Clin. Cancer Res. 37, 172 (2018).

13. Luo, L., Gao, Y. Q. \& Sun, X. F. Circular RNA ITCH suppresses proliferation and promotes apoptosis in human epithelial ovarian cancer cells by sponging miR-10a-alpha. Eur. Rev. Med. Pharm. Sci. 22, 8119-8126 (2018).

14. Wang, M., Chen, B., Ru, Z. \& Cong, L. CircRNA circ-ITCH suppresses papillary thyroid cancer progression through miR-22-3p/CBL/beta-catenin pathway. Biochem. Biophys. Res. Commun. 504, 283-288 (2018).

15. Gu, X., Li, M., Jin, Y., Liu, D. \& Wei, F. Identification and integrated analysis of differentially expressed IncRNAs and circRNAs reveal the potential ceRNA networks during PDLSC osteogenic differentiation. BMC Genet. 18, 100 (2017).

16. Shi, K. et al. MicroRNA-214 suppresses osteogenic differentiation of $\mathrm{C} 2 \mathrm{C} 12$ myoblast cells by targeting Osterix. Bone 55, 487-494 (2013).

17. Yao, S. et al. MicroRNA-214 suppresses osteogenic differentiation of human periodontal ligament stem cells by targeting ATF4. Stem Cells Int. 2017, 3028647 (2017)

18. Guo, Y., Li, L., Gao, J., Chen, X. \& Sang, Q. miR-214 suppresses the osteogenic differentiation of bone marrow-derived mesenchymal stem cells and these effects are mediated through the inhibition of the JNK and p38 pathways. Int J. Mol. Med. 39, 71-80 (2017).

19. Li, F., Ma, K., Sun, M. \& Shi, S. Identification of the tumor-suppressive function of circular RNA ITCH in glioma cells through sponging miR-214 and promoting linear ITCH expression. Am. J. Transl. Res. 10, 1373-1386 (2018).
20. Halder, G. \& Johnson, R. L. Hippo signaling: growth control and beyond. Development 138, 9-22 (2011).

21. Zhao, B., Li, L., Lei, Q. \& Guan, K. L. The Hippo-YAP pathway in organ size control and tumorigenesis: an updated version. Genes Dev. 24, 862-874 (2010).

22. Guo, L. et al. Kindlin-2 regulates mesenchymal stem cell differentiation through control of YAP1/TAZ. J. Cell Biol. 217, 1431-1451 (2018).

23. Pan, J. X. et al. YAP promotes osteogenesis and suppresses adipogenic differentiation by regulating beta-catenin signaling. Bone Res. 6, 18 (2018).

24. Li, X. et al. Stimulation of Piezo1 by mechanical signals promotes bone anabolism. Elife 8, e49631 (2019).

25. Mohamed-Ahmed, S. et al. Adipose-derived and bone marrow mesenchymal stem cells: a donor-matched comparison. Stem Cell Res. Ther. 9, 168 (2018).

26. Luo, Y. et al. Runx1 regulates osteogenic differentiation of BMSCs by inhibiting adipogenesis through Wnt/beta-catenin pathway. Arch. Oral. Biol. 97, 176-184 (2019).

27. Cho, C. S. et al. Anti-osteoporotic effects of mixed compositions of extracellular polymers isolated from Aureobasidium pullulans and Textoria morbifera in ovariectomized mice. BMC Complement. Alter. Med. 18, 295 (2018).

28. Chen, S., Zheng, Y., Zhang, S., Jia, L. \& Zhou, Y. Promotion effects of miR-375 on the osteogenic differentiation of human adipose-derived mesenchymal stem cells. Stem Cell Rep. 8, 773-786 (2017).

29. He, S. et al. LnCRNA ODIR1 inhibits osteogenic differentiation of hUC-MSCs through the FBXO25/H2BK120ub/H3K4me3/OSX axis. Cell Death Dis. 10, 947 (2019).

30. Tang, Y. et al. MicroRNA-99a is a novel regulator of KDM6B-mediated osteogenic differentiation of BMSCs. J. Cell Mol. Med. 22, 2162-2176 (2018).

31. Eslaminejad, M. B., Fani, N. \& Shahhoseini, M. Epigenetic regulation of osteogenic and chondrogenic differentiation of mesenchymal stem cells in culture. Cell J. 15, 1-10 (2013).

32. Sun, M. et al. The regulatory roles of microRNAs in bone remodeling and perspectives as biomarkers in osteoporosis. Biomed. Res. Int. 2016, 1652417 (2016).

33. Chen, X. et al. CircRNA_28313/miR-195a/CSF1 axis modulates osteoclast differentiation to affect OVX-induced bone absorption in mice. RNA Biol. 16, 1249-1262 (2019).

34. Ouyang, Z. et al. CircRNA hsa_circ_0074834 promotes the osteogenesisangiogenesis coupling process in bone mesenchymal stem cells (BMSCs) by acting as a ceRNA for miR-942-5p. Cell Death Dis. 10, 932 (2019).

35. Yin, Q., Wang, J., Fu, Q., Gu, S. \& Rui, Y. CircRUNX2 through has-miR-203 regulates RUNX2 to prevent osteoporosis. J. Cell Mol. Med. 22, 6112-6121 (2018).

36. Qian, D. Y. et al. Differential circRNA expression profiles during the BMP2induced osteogenic differentiation of MC3T3-E1 cells. Biomed. Pharmacother 90, 492-499 (2017).

37. Li, Y., Ge, Y. Z., Xu, L. \& Jia, R. Circular RNA ITCH: A novel tumor suppressor in multiple cancers. Life Sci. 254, 117176 (2020).

38. Ren, $\mathrm{C}$. et al. The circular RNA circ-ITCH acts as a tumour suppressor in osteosarcoma via regulating miR-22. Artif. Cells Nanomed. Biotechnol. 47, 3359-3367 (2019)

39. Wang, C. G. et al. Pseudogene PTENP1 sponges miR-214 to regulate the expression of PTEN to modulate osteoclast differentiation and attenuate osteoporosis. Cytotherapy 22, 412-423 (2020).

40. Wang, C. G. et al. LncRNA KCNQ1OT1 promoted BMP2 expression to regulate osteogenic differentiation by sponging miRNA-214. Exp. Mol. Pathol. 107 77-84 (2019).

41. Li, S. et al. Circular RNA 101368/miR-200a axis modulates the migration of hepatocellular carcinoma through HMGB1/RAGE signaling. Cell Cycle 17, 2349-2359 (2018).

42. Li, H. et al. CircRNA CBL.11 suppresses cell proliferation by sponging miR-6778$5 p$ in colorectal cancer. BMC Cancer 19, 826 (2019).

43. Zhu, S. et al. miR-1827 inhibits osteogenic differentiation by targeting IGF1 in MSMSCs. Sci. Rep. 7, 46136 (2017).

44. Lorthongpanich, C. et al. YAP as a key regulator of adipo-osteogenic differentiation in human MSCs. Stem Cell Res. Ther. 10, 402 (2019). 